\section{Unlike climate science, GM is full of uncertainties}

SIR — Greenpeace has been invited by David Dennis, in Correspondence, to reconsider our opposition to genetically modified (GM) crops in the light of our support for the consensus on climate science ("Activists should accept mainstream view of GM" Nature 435, 561; 2005). There are two factors to consider when deciding to support any apparent scientific consensus.

First, what was the process of arriving at the current mainstream position? In the case of climate change, uncertainties over the physics, measurements, modelling and historical data have generally (although sometimes erratically) tended to be resolved. In the case of GM, further investigation of genomes and gene function has led to new insights, such as alternative splicing mechanisms and the regulatory roles played by RNAi and chromatin packing, which question the fundamental understanding of gene regulation and control. This is demonstrated by the hedging on certainties in the UK government's GM science panel review in 2003, which was a far cry from the certainties expressed in the mid-1990s.

Second, as an environmental-protection organization, it should come as no surprise that we interpret scientific uncertainty in favour of environmental protection. Anyone who interprets a given level of uncertainty to propose a policy action must be imposing their values, even if that means advocating “do nothing now except more research".

Whereas we are dear about our values, one might wonder what values are espoused by those, especially in the United States, who support GM organisms but reject the evidence of climate change.

Douglas Parr

Greenpeace UK, Canonbury Villas, London N12PN, UK

\section{Leave $\mathbf{G M}$ analysis to the relevant scientists}

SIR — David Dennis, in Correspondence, claims that "an overwhelming majority of plant geneticists, biochemists and molecular biologists have endorsed the use and safety" of genetically modified (GM) crops ("Activists should accept mainstream view of $\mathrm{GM}^{*}$ Nature 435,$\left.561 ; 2005\right)$. I question the validity of that claim.

Assessing the potential environmental and/or economical consequences of using GM crops - such as their impact on soil fauna or on non-target organisms - requires analysis in crop fields and in the natural environment, working on relevant objects, at the relevant scale.
As questions about the use and safety of GM crops concern primarily environmental science, statements by biochemists and molecular biologists, who deal with simplified biological systems, at small scales, only add to the problem of misinformation and lead to an increase in concern about GM crops.

\section{"Statements by scientists who deal} with simplified biological systems, at small scales, only add to the problem of misinformation."

\section{- Denis Couvet}

Perhaps the public would be less worried if it was the overwhelming majority of environmental scientists who felt confident about the use and safety of GM crops.

Denis Couvet

Department of Ecology,

Muséum National d'Histoire Naturelle,

55 rue Buffon, 75005 Paris, France

\section{Compensation for climate change must meet needs}

SIR - Sujatha Byravan and Sudhir Chella Rajan, in Correspondence ("Immigration could ease dimate-change impact" Nature 434, 435; 2005), argue that major greenhousegas emitters should provide compensation for the impacts of climate change.

We believe that compensation is on the cards. We also believe there will eventually be the science to accurately establish liability for those impacts (see M. R. Allen and R. Lord, Nature 432, 551-552; 2004). But the nature of compensation will always remain contested. The notion that migration is a sustainable adaptation strategy for future climate change ignores the fact that patterns of migration are strongly based on social networks and cultural links. Legislating for flows of people may simply not appeal to migrants.

New Zealand's creation of the Pacific Access Category, in response to concerns about climate change, is an instructive example. The scheme allows for up to 75 people from Tuvalu to migrate each year, but since it began in July 2002, fewer than half the places available have been filled. This possibly suggests that even in Tuvalu, where there is widespread concern about dimate change, people are not eager to leave their homeland. This example points to the need for policies and measures that help people adapt to climate change, in order to lead the kind of lives they value in the places where they belong, rather than to encourage migration.

The United Nations Framework Convention on Climate Change (UNFCCC) is developing mechanisms to help vulnerable countries adapt to climate change. However, these mechanisms are not the obligatory compensation transfers that Byravan and Rajan espouse. Indeed, the prospect of enforceable migration threatens voluntary processes, such as the UNFCCC, as they may deter large greenhouse-gas polluters from participating in the process.

We believe that promoting and funding activities that enhance in situ adaptation for vulnerable populations is a more practicable and equitable approach than migrationbased compensation strategies.

W. Neil Adger ${ }^{\star}$, Jon Barnett

*Tyndall Centre for Climate Change Research, University of East Anglia, Norwich NR4 7TJ, UK †School of Anthropology, Geography and Environmental Studies, University of Melbourne, Melbourne, Victoria 3010, Australia

\section{There's more to a colourful life than simply sex}

SIR - Rolf Hoekstra in News and Views ("Why sex is good" Nature 434, 571; 2005) surely overstates the contribution of sexual reproduction to the aesthetic appeal of nature. He assumes that complex organisms similar to flowering plants, insects and peacocks would evolve, but that without sex the world would be drab and colourless. However, asexually reproducing plants would probably still take advantage of animals for spore dispersal, producing fruits or other rewards (advertised by special structures) as 'payment.' Indeed it is likely that our colour vision developed in order to detect ripe fruits, which have little to do with sex and a lot to do with seed dispersal.

Similarly, although it is true that many gaudy avian displays are aimed at mate attraction, there are many brightly coloured animals whose decoration serves as a threat or warning. Colonial bees, wasps and many butterflies and moths all bear distinctive and colourful markings discouraging interference.

Sexual reproduction, in maintaining genetic heterogeneity within a population, is clearly a major mechanism by which species survive catastrophes and adapt to the subsequent conditions. However, the pro-sex lobby always seems to downplay the importance of asexual reproduction to evolution in stable environments. Apomictic organisms (not strictly asexual of course), such as dandelions, can develop localized populations accumulating mutations that render them distinct from other members of their clade. This kind of diversification is rarely seen in sexually reproducing populations unless they are subjected to selective pressure.

Sex is good, but it ain't everything. Paul Kenton

University of Wales, Aberystwyth SY23 3DA, UK 